\title{
Gallium Ga 68-Edotreotide
}

National Cancer Institute

\section{Source}

National Cancer Institute. Gallium Ga 68-Edotreotide. NCI Thesaurus. Code C101369.

A radioconjug ate consisting of the octreotide derivative edotreotide labeled with gallium 68 (Ga-68) with potential application in somatostatin receptor (SST R) imaging in conjunction with positron emission tomography (PET). Similar to octreotide, gallium Ga 68-edotreotide binds to SSTRs, especially type 2 receptors, present on the cell membranes of many types of neuroendocrine tumor cells and their metastases, thereby allowing for imaging of SST R-expressing cells upon PET. Gallium Ga 68-edotreotide is produced by substituting tyrosine for phenylalanine at the 3 position of the somatostatin analogue octreotide (Tyr3-octreotide or TOC) and chelating the substituted octreotide to Ga-68 via the macrocyclic chelating agent dodecanetetraacetic acid (DOTA). 